\title{
Electrically Forced Vibrations of Partially Electroded Rectangular Quartz Plate Piezoelectric Resonators
}

\author{
Hui Chen, Ji Wang and Jianke Du \\ Piezoelectric Device Laboratory, School of Mechanical Engineering and Mechanics, Ningbo University, Ningbo, \\ Zhejiang 315211, China
}

\author{
Jiashi Yang \\ Piezoelectric Device Laboratory, School of Mechanical Engineering and Mechanics, Ningbo University, Ningbo, \\ Zhejiang 315211, China \\ Department of Mechanical and Materials Engineering, the University of Nebraska-Lincoln, Lincoln, NE 68588- \\ 0526, USA
}

(Received 3 October 2014; accepted 16 February 2015)

\begin{abstract}
We performed a theoretical analysis on electrically forced, coupled thickness-shear, and flexural vibrations of a rectangular AT-cut quartz resonator, which is electroded in its central part only. Mindlin's first-order theory for piezoelectric plates is used and simplified by the flexure-twist approximation due to Mindlin and Spencer. An analytical solution is then obtained. The admittance of the resonator under a time-harmonic driving voltage, which is an important property in resonator design, is calculated and examined. The mode shapes at resonances are also presented and discussed. It is found that for a perfectly symmetric resonator only cylindrical modes depending on one of the two in-plane spatial coordinates can be excited by the applied voltage. This supports the conventional approximate analyses based on the assumption of cylindrical deformations.
\end{abstract}

\section{INTRODUCTION}

Piezoelectric crystals are widely used to make resonators for time-keeping, frequency generation and operation, telecommunication, and sensing. Quartz is the most widely used crystal for resonator applications. Due to material anisotropy and electromechanical coupling, a theoretical analyses of crystal resonators presents considerable mathematical challenges. Since many piezoelectric resonators operate with thicknessshear (TSh) vibration modes of plates, ${ }^{1-4}$ Mindlin et al. ${ }^{1-4}$ developed and refined the two-dimensional (2-D) equations for motions of piezoelectric plates. The 2-D equations lead to many theoretical results useful for the understanding and design of piezoelectric resonators. For example, the behaviors of cylindrical TSh motions or the so-called straight-crested waves depending on one of the two in-plane spatial variables of the crystal plates only are now reasonably well understood $.^{5-7} \mathrm{Fi}-$ nite element procedures based on the 2-D plate equations were also developed. ${ }^{8,9}$ More references can be found in a review article by Wang and Yang. ${ }^{10}$

Even with the 2-D plate equations, there are practically important situations for which the available theoretical results are very limited, which means there is a lack of basic understanding. This is because real crystal resonators actually used in various electronic equipment are with partial electrodes. When using the 2-D plate equation to analyze these resonators, the electroded and unelectroded regions need to be treated separately using slightly different equations with continuity conditions between them. This is complicated and sometimes it can be impractical or impossible, except in the case of cylindrical motions where the fields vary along one of the two in-plane directions of a plate only. When the vibration varies in both of the in-plane directions of a plate, there are very few theoretical results on partially electroded resonators from the 2-D equations, especially for electrically forced vibrations.

For coupled TSh and flexural motions of a plate, a procedure called flexure-twist approximation was developed ${ }^{11}$ for anisotropic elastic plates, which describes a plate sheardeformable in one of the two in-plane directions of the plate and is shear-rigid in the perpendicular direction. The flexuretwist approximation was later extended to the case of piezoelectric plates. ${ }^{12}$ This approximation simplifies the 2-D plate equations significantly, with which the free vibration solution of a rectangular quartz plate was obtained by Mindlin and Spencer ${ }^{11}$ which provides basic information on the resonant frequencies and mode shapes of the plate. However, the understanding of the behaviors of a partially electroded, rectangular resonator in electrically forced vibrations is still very much in need. This is because resonators are used as elements of electrical circuits of alternating currents. Two basic properties of a resonator, its resonant frequency and admittance, are both of primary interest for circuit analyses. A free vibration analysis can only produce resonant frequencies and modes. The admittance of a resonator can only be obtained from an electrically forced vibration analysis. Recently, there has been growing interest in forced vibration analyses of piezoelectric plates and the computation of resonator admittance, ${ }^{13-15}$ which are for cylindrical motions. In this paper, we use Mindlins first- 


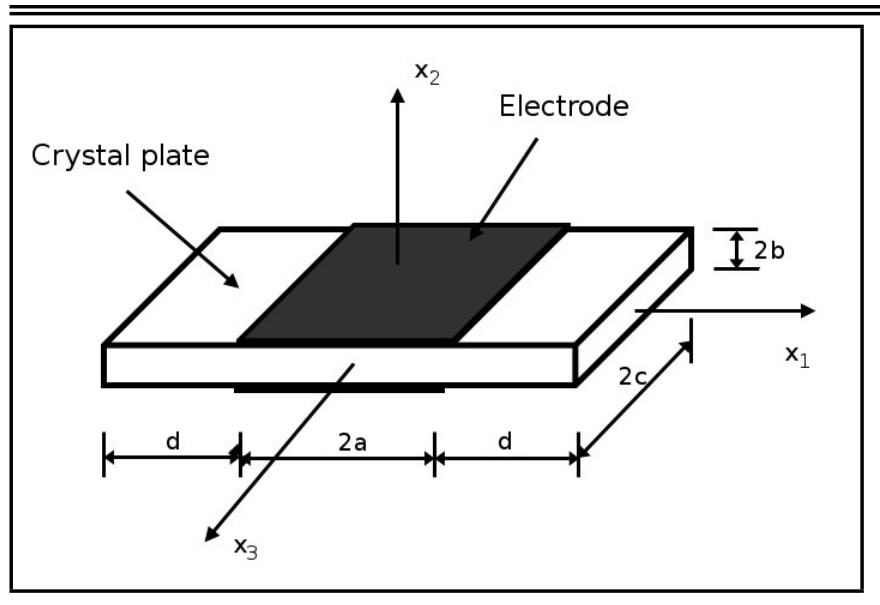

Figure 1. A partially electroded piezoelectric resonator of monoclinic crystals.

order plate theory ${ }^{3}$ simplified by the flexure-twist approximation $^{11,12}$ to analyze electrically forced, coupled TSh and flexural vibrations of a partially electroded rectangular piezoelectric resonator of monoclinic crystals. This includes the commonly used rotated Y-cut quartz and langasite crystal plates as special cases.

\section{GOVERNING EQUATIONS}

Consider the partially electroded plate of monoclinic crystals shown in Fig. 1. Its thickness and mass density are $2 b$ and $\rho$, respectively. The thickness and mass density of the electrodes are $2 b^{\prime}$ and $\rho^{\prime}$, respectively. The mass ratio between the electrodes and the crystal plate is $R=2 b^{\prime} \rho^{\prime} /(b \rho)$. Mindlin's 2-D plate equations are slightly different for electroded and unelectroded plates. We begin with an electroded plate. For TSh resonators the dominating displacement is $u_{1}^{(1)}\left(x_{1}, x_{3}, t\right)$, which is usually coupled to the unwanted flexural displacement $u_{2}^{(0)}\left(x_{1}, x_{3}, t\right)$. Under the flexure-twist approximation, the TSh and flexural motions are governed by ${ }^{11}$

$$
\begin{gathered}
T_{12,1}^{(0)}+T_{32,3}^{(0)}=2 b \rho(1+R) \ddot{u}_{2}^{(0)} ; \\
T_{11,1}^{(1)}+T_{31,3}^{(1)}-T_{21}^{(0)}=\frac{2 b^{3}}{3} \rho(1+3 R) \ddot{u}_{1}^{(1)} ;
\end{gathered}
$$

where the relevant plate resultants describing shear forces and bending/twisting moments take the following form: ${ }^{12}$

$$
\begin{gathered}
T_{12}^{(0)}=2 b\left[\bar{\kappa}_{1}^{2} c_{66}\left(u_{2,1}^{(0)}+u_{1}^{(1)}\right)+\kappa_{1} e_{26} \varphi^{(1)}\right] \\
T_{32}^{(0)}=T_{13,1}^{(1)}=\frac{2 b^{3}}{3}\left[\gamma_{55}\left(u_{1,31}^{(1)}-u_{2,311}^{(0)}\right)+\psi_{35} \varphi_{, 31}^{(1)}\right] \\
T_{11}^{(1)}=\frac{2 b^{3}}{3}\left(\gamma_{11} u_{1,1}^{(1)}+\psi_{11} \varphi_{, 1}^{(1)}\right) \\
T_{31}^{(1)}=\frac{2 b^{3}}{3}\left[\gamma_{55}\left(u_{1,3}^{(1)}-u_{2,31}^{(0)}\right)+\psi_{35} \varphi_{, 3}^{(1)}\right]
\end{gathered}
$$

In Eq. (2), the plate material constants are related to the usual three-dimensional elastic constants $c_{p q}$ (or $s_{p q}$ ), piezoelectric constants $e_{i p}$, and dielectric constants $\varepsilon_{i j}$ through

$$
\gamma_{11}=s_{33} /\left(s_{11} s_{33}-s_{13}^{2}\right)
$$

$$
\begin{gathered}
\gamma_{55}=c_{55}-c_{56}^{2} / c_{66}=1 / s_{55} ; \\
\psi_{11}=d_{11} \gamma_{11}+d_{13} \gamma_{13} ; \\
\psi_{35}=e_{35}-e_{36} c_{56} / c_{66}=d_{35} \gamma_{55} .
\end{gathered}
$$

$\bar{\kappa}_{1}$ is a correction factor given by ${ }^{11,12}$

$$
\begin{gathered}
\bar{\kappa}_{1}^{2}=\frac{\pi^{2}}{12}\left(1+R-\frac{8}{\pi^{2}} k_{26}^{2}\right) ; \\
k_{26}^{2}=\frac{e_{26}^{2}}{\varepsilon_{22} \hat{c}_{66}} ; \\
\hat{c}_{66}=c_{66}+\frac{e_{26}^{2}}{\varepsilon_{22}} .
\end{gathered}
$$

When there is an alternating voltage $V \exp (i \omega t)$ applied across the electrodes, the $\varphi^{(1)}$ in Eq. (1) is related to the voltage by ${ }^{3}$

$$
\varphi^{(1)}=\frac{1}{2 b} V \exp (i \omega t)
$$

The substitution of Eqs. (2) and (6) into Eq. (1) gives

$$
\begin{array}{r}
\bar{\kappa}_{1}^{2} c_{66}\left(u_{2,11}^{(0)}+u_{1,1}^{(1)}\right)+\frac{b^{2}}{3} \gamma_{55}\left(u_{1,313}^{(1)}-u_{2,3113}^{(0)}\right)= \\
=\rho(1+R) \ddot{u}_{2}^{(0)}
\end{array}
$$

We consider steady-state, time-harmonic motions. All fields have the same time-harmonic factor. The free charge $Q_{e}$ on the upper electrode and the current $I$ flowing into this electrode can be calculated from ${ }^{12}$

$$
\begin{gathered}
Q_{e}=-\int_{-a}^{a} \int_{-c}^{c} D_{2} d x_{1} d x_{3} \\
I=\dot{Q}_{e}=i \omega Q_{c}
\end{gathered}
$$

where

$$
D_{2} \cong \frac{D_{2}^{(0)}}{2 b}=\bar{\kappa}_{1} e_{26}\left(u_{2,1}^{(0)}+u_{1}^{(1)}\right)-\varepsilon_{22} \phi^{(1)} .
$$

Then the frequency-dependent admittance of the resonator is given by

$$
A=I / V
$$

Quartz is a material with very weak piezoelectric coupling. In an unelectroded plate the electric field is small because it is produced by mechanical fields through the small piezoelectric coupling instead of a driving voltage. Therefore, we neglect the small electric field and treat an unelectroded plate as an anisotropic elastic plate. This approximation is frequently used in the analysis of quartz resonators. ${ }^{11}$ Therefore, for an unelectroded plate, formally Eqs. (1), (2), and (7) still apply, with the 


$$
\begin{gathered}
\left|\begin{array}{cc}
3 \rho(1+R) \omega^{2}-3 \bar{\kappa}_{1}^{2} c_{66} \bar{\xi}^{2}-\gamma_{55} b^{2} \bar{\xi}^{2} \zeta^{2} & \gamma_{55} b^{2} \bar{\xi} \zeta^{2}-3 \bar{\kappa}_{1}^{2} c_{66} \bar{\xi} \\
\gamma_{55} b^{2} \bar{\xi} \zeta^{2}-3 \bar{\kappa}_{1}^{2} c_{66} \bar{\xi} & \rho \omega^{2}(1+3 R) b^{2}-\gamma_{11} \bar{\xi}^{2} b^{2}-\gamma_{55} \zeta^{2} b^{2}-3 \bar{\kappa}_{1}^{2} c_{66}
\end{array}\right|=0 ; \\
\left|\begin{array}{cc}
3 \rho \omega^{2}-3 \kappa_{1}^{2} c_{66} \xi^{2}-\gamma_{55} b^{2} \xi^{2} \zeta^{2} & \gamma_{55} b^{2} \xi \zeta^{2}-3 \kappa_{1}^{2} c_{66} \xi \\
\gamma_{55} b^{2} \xi \zeta^{2}-3 \kappa_{1}^{2} c_{66} \xi & \rho \omega^{2} b^{2}-\gamma_{11} \xi^{2} b^{2}-\gamma_{55} \xi^{2} b^{2}-3 \kappa_{1}^{2} c_{66}
\end{array}\right|=0 ; \\
\left|\begin{array}{cc}
3 \rho \omega^{2}-3 \kappa_{1}^{2} c_{66} \xi^{2}-\gamma_{55} b^{2} \xi^{2} \zeta^{2} & -\gamma_{55} b^{2} \xi \zeta^{2}+3 \kappa_{1}^{2} c_{66} \xi \\
-\gamma_{55} b^{2} \xi \zeta^{2}+3 \kappa_{1}^{2} c_{66} \xi & \rho \omega^{2} b^{2}-\gamma_{11} \zeta^{2} b^{2}-\gamma_{55} \zeta^{2} b^{2}-3 \kappa_{1}^{2} c_{66}
\end{array}\right|=0 ;
\end{gathered}
$$

mass ration $\mathrm{R}=0$, the applied voltage $V=0$ or $\phi^{(1)}=0$, and the correction factor $\kappa_{1}^{2}=\pi^{2} / 12$.

The boundary conditions for the free edges of the plate in Fig. 1 are $^{11}$

$$
\begin{gathered}
T_{12}^{(0)}+T_{31,3}^{(1)}=0 ; \\
T_{11}^{(1)}=0 ; \\
x_{1}= \pm(a+d) ; \\
T_{31}^{(1)}=0 ; \\
x_{3}= \pm c .
\end{gathered}
$$

At the junctions between the electroded and unelectroded parts in Fig. 1, we have the following continuity conditions: ${ }^{11}$

$$
\begin{gathered}
{\left[u_{2}^{(0)}\right]=0 ;} \\
{\left[u_{1}^{(1)}\right]=0 ;} \\
{\left[T_{11}^{(1)}\right]=0 ;} \\
{\left[T_{12}^{(0)}+T_{31,3}^{(1)}\right]=0 ;} \\
x_{1}= \pm a ;
\end{gathered}
$$

where the square brackets represent the jumps of the fields across the junctions.

\section{FORCED VIBRATION SOLUTION}

From the symmetries present in the structure in Fig. 1, the electrically excited TSh vibration is symmetric about both the $x_{1}$ and $x_{3}$ axes. We only need to consider the right half of the plate with $x_{1}>0$. In the electroded region with $0<x_{1}<a$, since Eq. (7) is inhomogeneous, its general solution can be written as the sum of the general solution of the corresponding homogeneous equation and a particular solution, i.e.,

$$
\begin{gathered}
u_{2}^{(0)}=\sum_{j=1}^{2}\left(A_{j} \sin \bar{\xi}_{j} x_{1}\right) \cos \zeta x_{3} \\
u_{1}^{(1)}=\sum_{j=1}^{2}\left(\alpha_{j} A_{j} \cos \bar{\xi}_{j} x_{1}\right) \cos \zeta x_{3}+\tilde{u}_{1}^{(1)}
\end{gathered}
$$

where the particular solution is given by

$$
\tilde{u}_{1}^{(1)}=\frac{3 \bar{\kappa}_{1} e_{26}}{\rho(1+3 R) \omega^{2} b^{2}-3 \bar{\kappa}_{1}^{2} c_{66}} \frac{V}{2 b} .
$$

In Eq. (13), $A_{j}$ are undetermined constants. $\bar{\xi}_{j}^{2}$ are the two roots of the Eq. (15) (see on top of the page), and

$$
\alpha_{j}=-\frac{3 \rho(1+R) \omega^{2}-3 \bar{\kappa}_{1}^{2} c_{66} \bar{\xi}_{j}^{2}-\gamma_{55} b^{2} \bar{\xi}_{j}^{2} \zeta^{2}}{\gamma_{55} b^{2} \bar{\xi}_{j} \zeta^{2}-3 \bar{\kappa}_{1}^{2} c_{66} \bar{\xi}_{j}} .
$$

In Eq. (13), the vibration varies along both the $x_{1}$ and $x_{3}$ directions. The variation along $x_{3}$ is described by $\cos \zeta x_{3}$ with $\zeta=p \pi /(2 c)$ where $p=0,2,4, \cdots .{ }^{11}$ Thus, the boundary conditions at $x_{3}= \pm c$ in (11b) are satisfied. $\cos \zeta x_{3}$ with $p=0,2,4, \cdots$ form a complete set of base functions for symmetric function of $x_{3}$ over $-c<x_{3}<c$. Strictly speaking, a summation with respect to $p$ resenting a series should be used in Eq. (13). Since every term of the series satisfies the boundary conditions at $x_{3}= \pm c$ in Eq. (11), we are treating the series term by term, which is simpler in writing. In general the particular solution in Eq. (14) should also be expressed as a series of $\cos \zeta x_{3}$, but in our case, this series has only one constant term corresponding to $p=0$, which is the particular solution itself.

For the unelectroded part of the plate with $a<x_{1}<a+d$, the governing equations corresponding to Eq. (7) are homogeneous. The general solution can be written as

$$
\begin{gathered}
u_{2}^{(0)}=\sum_{j=1}^{2}\left(B_{j} \sin \xi_{j} x_{1}+C_{j} \cos \xi_{j} x_{1}\right) \cos \zeta x_{3} ; \\
u_{1}^{(1)}=\sum_{j=1}^{2}\left(\beta_{j} B_{j} \cos \xi_{j} x_{1}+\gamma_{j} C_{j} \sin \xi_{j} x_{1}\right) \cos \zeta x_{3} .
\end{gathered}
$$

In Eq. (17), $B_{j}$ are undetermined constants and the related $\xi_{j}^{2}$ and $\beta_{j}$ are determined by Eq. (18) (see on top of the page) and

$$
\beta_{j}=-\frac{3 \rho \omega^{2}-3 \kappa_{1}^{2} c_{66} \xi_{j}^{2}-\gamma_{55} b^{2} \xi_{j}^{2} \zeta^{2}}{\gamma_{55} b^{2} \xi_{j} \zeta^{2}-3 \kappa_{1}^{2} c_{66} \xi_{j}} .
$$

Similarly, $C_{j}$ are also undetermined constants and the corresponding $\xi_{j}^{2}$ and $\gamma_{j}$ are determined by Eq. (20) (see on top of the page) and

$$
\gamma_{j}=\frac{3 \rho \omega^{2}-3 \kappa_{1}^{2} c_{66} \xi_{j}^{2}-\gamma_{55} b^{2} \xi_{j}^{2} \zeta^{2}}{\gamma_{55} b^{2} \xi_{j} \zeta^{2}-3 \kappa_{1}^{2} c_{66} \xi_{j}} .
$$

The substitution of Eqs. (13) and (17) into the two boundary conditions at $x_{1}=a+d$ in (11a) and the four continuity conditions at $x_{1}=a$ in Eq. (12) leads to

$$
\begin{aligned}
2 b \kappa_{1}^{2} c_{66} & \sum_{j=1}^{2}\left[\left(\xi_{j}+\beta_{j}\right) B_{j} \cos \xi_{j}(a+d)+\right. \\
& \left.+\left(\gamma_{j}-\xi_{j}\right) C_{j} \sin \xi_{j}(a+d)\right]=0
\end{aligned}
$$




$$
\begin{gathered}
\sum_{j=1}^{2} \xi_{j}\left[-\beta_{j} B_{j} \sin \xi_{j}(a+d)+\gamma_{j} C_{j} \cos \xi_{j}(a+d)\right]=0 \\
\left.\sum_{j=1}^{2}\left(A_{j} \sin \bar{\xi}_{j} a\right)=\sum_{j=1}^{2}\left(B_{j} \sin \xi_{j} a+C_{j} \cos \xi_{j} a\right) ; \quad \text { (22) }\right) \\
\sum_{j=1}^{2}\left(\alpha_{j} A_{j} \cos \bar{\xi}_{j} a\right)+\tilde{u}_{1}^{(1)}= \\
\sum_{j=1}^{2}\left(\beta_{j} B_{j} \xi_{j} \sin \xi_{j} a+\gamma_{j} C_{j} \sin \xi_{j} a\right) ; \\
-\sum_{j=1}^{2}\left(\alpha_{j} \bar{\xi}_{j} A_{j} \sin \bar{\xi}_{j} a\right)= \\
=\sum_{j=1}^{2}\left(-\beta_{j} B_{j} \xi_{j} \sin \xi_{j} a+\gamma_{j} C_{j} \xi_{j} \cos \xi_{j} a\right) ; \\
2 b \bar{\kappa}_{1}^{2} c_{66} \sum_{j=1}^{2}\left[\left(\bar{\xi}_{j}+\alpha_{j}\right) A_{j} \cos \bar{\xi}_{j} a+\tilde{u}_{1}\right]+\kappa_{1} e_{26} V= \\
=2 b \kappa_{1}^{2} c_{66} \sum_{j=1}^{2}\left[\left(\xi_{j}+\beta_{j}\right) B_{j} \cos \xi_{j} a+\left(\gamma_{j}-\xi_{j}\right) C_{j} \sin \xi_{j} a\right] ;
\end{gathered}
$$

which are six linear algebraic equations for the six undetermined constants $A_{j}, B_{j}$ and $C_{j}$. These equations are inhomogeneous and driven by $V$. They are solved on a computer using MATLAB.

\section{NUMERICAL RESULTS AND DISCUSSION}

As a numerical example, consider an AT-cut quartz plate resonator with a typical thickness described by $b=0.3173 \mathrm{~mm}$. When the small piezoelectric coupling in quartz is neglected, an infinite and unelectroded plate with such a thickness has the following fundamental TSh frequency:

$$
\omega_{0}=\frac{\pi}{2 b} \sqrt{\frac{c_{66}}{\rho}}=16.384 \mathrm{MHz}
$$

which will be used as a frequency unit. In our numerical example, the electrode/plate mass ratio $R=0.005$ is fixed except in Fig. 5. Both $a=15 b=4.7591 \mathrm{~mm}$ and $d=10 b=3.1727 \mathrm{~mm}$ are fixed except in Fig. 6. Material damping is introduced by allowing the relevant elastic constants to assume complex values, which can represent viscous damping. In our numerical calculation, the elastic constants $c_{p q}$ is replaced by $c_{p q}\left(1+i Q^{-1}\right)$, where $Q$ is a real, positive, and large number. We fixed $Q=1000$ except in Fig. 7. This is a relatively small $Q$ for the material damping of quartz. A relatively small value of $Q$ is used as a representation of the total damping in the system.

We ploted the absolute value of the complex admittance calculated from Eq. (10) versus the driving frequency near the

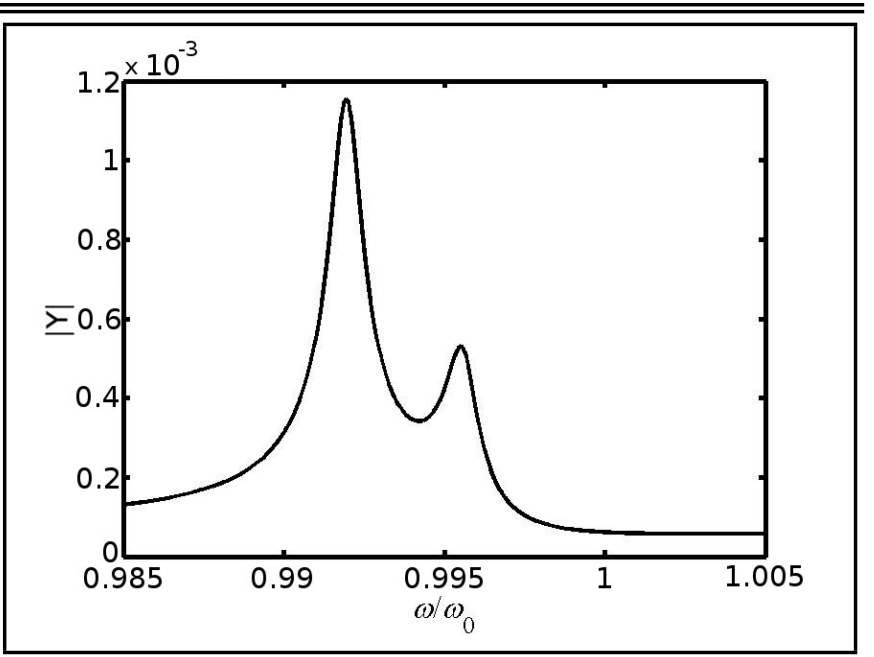

Figure 2. Admittance versus driving frequency near TSh resonances.

fundamental TSh frequency given by Eq. (23) in Fig. 2. There are two resonances ( 0.9920 and 0.9955 as normalized by (23)) in the frequency range of interest, i.e., when the normalized frequency is close to one from below because the electrode inertia lowers the resonant frequencies. Ideally, an isolated resonance peak is desired. However, due to coupling with flexure, there are usually secondary peaks near the main peak and what is shown in Fig. 2 is typical.

At the two resonances, the distributions of the TSh and flexural displacement components at the plate top surface are shown in Figs. 3 and 4, respectively. The TSh displacement is from the operating mode. It is much larger than the unwanted flexural displacement. This indicates a reasonably good resonator with a TSh dominated vibration and weak coupling to flexure. This coupling cannot be avoided totally because it is related to the wave reflections at the plate edges. The main TSh displacement is large in the electroded central region, and decays to almost zero near the plate edges. This is the so-called energy trapping of TSh modes by electrodes. Energy trapping is fundamental to resonator mounting which can be designed near the plate edges without affecting the vibration of the plate. We note that the small flexural displacement is sinusoidal and therefore is not trapped. This causes a small leakage of vibration energy into mounting which is part of the overall damping in resonators. We also note that the TSh displacement is a long wave and the flexural displacement is a relatively short wave.

Figure 5 shows the effect of the electrode/plate mass ratio $R$ on the admittance, which seems to be relatively simple. For thicker or heavier electrodes represented by larger values of $R$, the resonant frequencies become lower as expected because of more electrode inertia. At the same time, the magnitude of the admittance and the shapes of the two peaks do not seem to have changed.

If we vary the electrode length $2 a$ only and all other parameters, including the total length of the plate are kept the same as those used in Fig. 2, the result is shown in Fig. 6 in which larger electrodes result in large admittance as expected. Interestingly, while the location of the first peak does not have any noticeable change as the electrode length increases, the location of the second peak moves to the left in the process. This shows that the electrode length has a clear effect on mode coupling. 


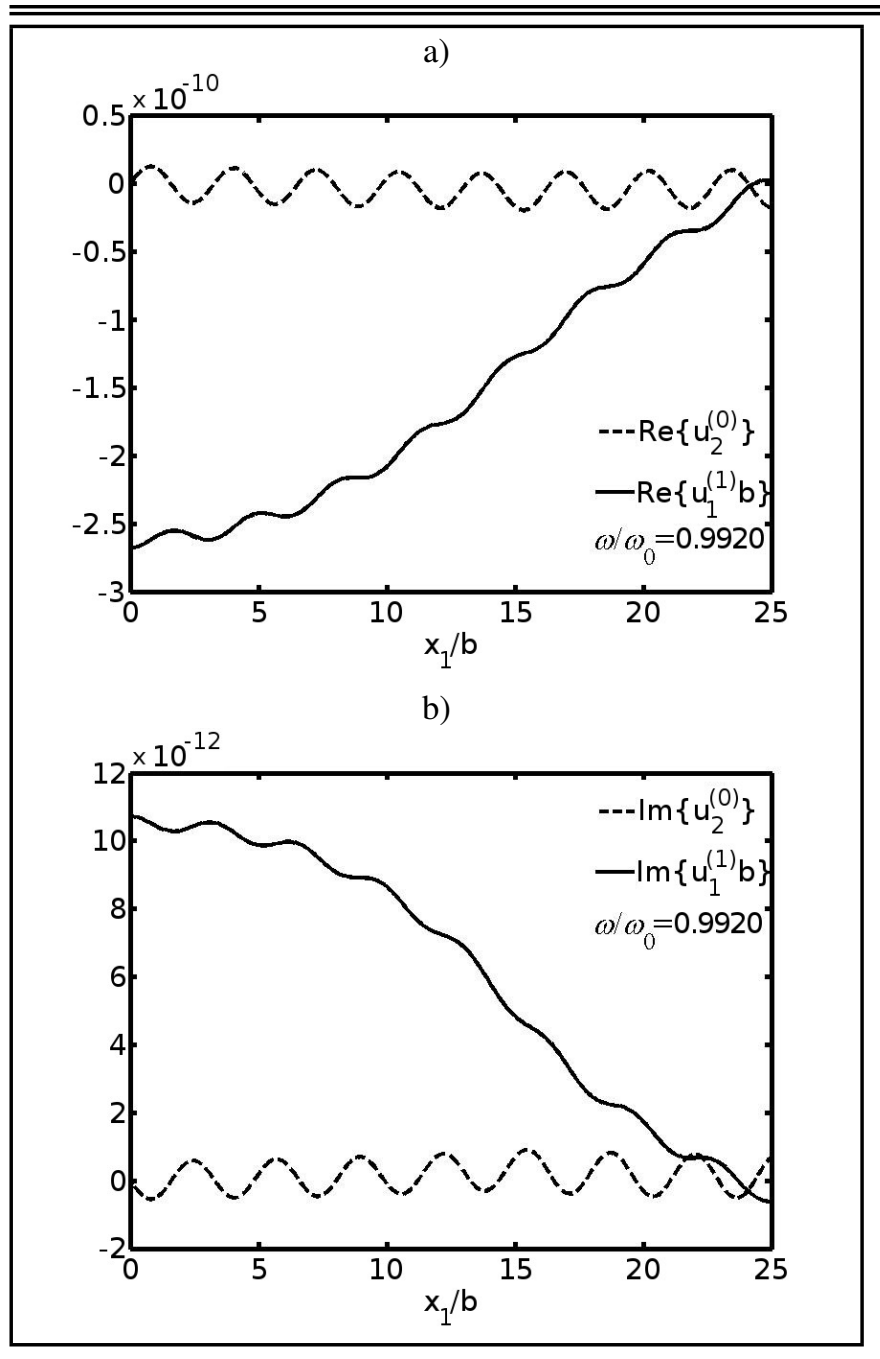

Figure 3. Plate surface displacement distributions at the first resonance in Fig. 2.

This is not surprising because the coupling between TSh and flexure is known to be sensitive to the plate length/thickness ratio. ${ }^{17}$ In a partially electroded plate as shown in Fig. 1, since the vibration is mainly in the electroded central region, what matters to mode coupling is the length of the electroded central region instead of the plate total length.

In Fig. 7, the damping described by $Q$ is varied while all other parameters are kept the same as those used for plotting Fig. 2. A larger value of $Q$ represents less damping in the system. This should imply a stronger mechanical resonance with a larger TSh deformation and more charges on the electrodes through piezoelectric coupling, and a larger admittance which is confirmed in Fig. 7.

\section{CONCLUSIONS}

The admittance is large at TSh resonances and is sensitive to system parameters. For electrically forced vibrations of partially electroded, rectangular, AT-cut quartz plates with free edges, the operating TSh mode is weakly coupled to the flexural mode. The TSh wave is long and is mainly trapped under the electrodes. The flexural wave is relatively short and not trapped. The in-plane TSh mode variation at resonances is mainly along $x_{1}$. In the $x_{3}$ direction, within the first- order Mindlin plate theory and its flexure-twist approximation, a)

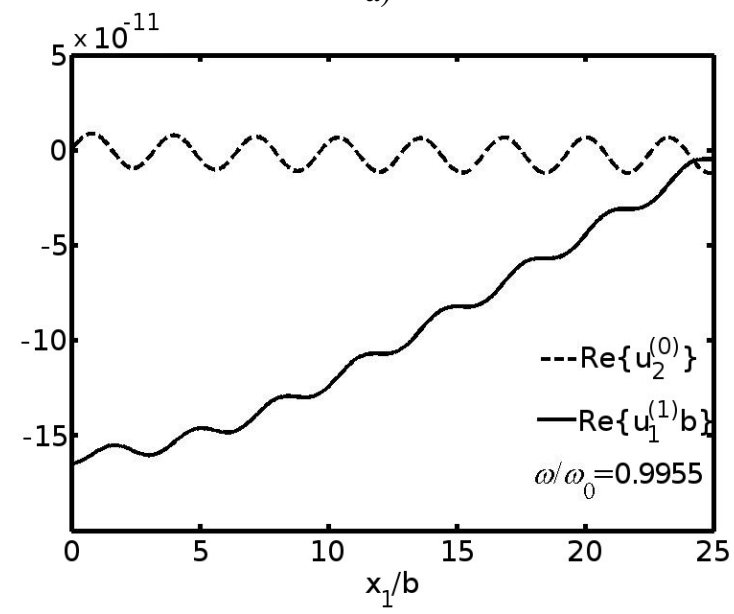

b)

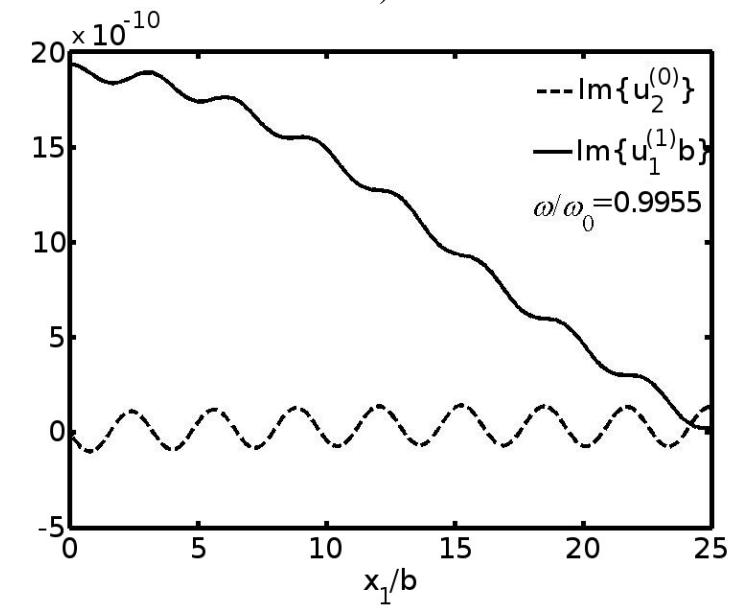

Figure 4. Plate surface displacement distributions at the second resonance in Fig. 2.

$x_{3}$ dependent modes cannot be excited by a thickness electric field in a structurally symmetric resonator. In this case, the usual analyses of cylindrical motions independent of $x_{3}$ is a very good approximation. In practice, structural imperfections may change the situation and introduce $x_{3}$ dependence. If the electrodes do not cover the entire width $2 c$ of the resonator and cover the central part along $x_{3}$ only, an $x_{3}$ dependent analysis becomes necessary, which remains mathematically challenging.

\section{ACKNOWLEDGEMENTS}

This work was supported by the National Natural Science Foundation of China (Nos. 11272161 and 11372145), the Y. K. Pao Visiting Professorship at Ningbo University, and the K. C. Wong Magana Fund through Ningbo University.

\section{REFERENCES}

1 Mindlin, R. D. High frequency vibrations of crystal plates, Quart. Appl. Math., 19 (1), 51-61, (1961).

2 Tiersten, H. F. and Mindlin, R. D. Forced vibrations of piezoelectric crystal plates, Quart. Appl. Math., 20 (2), 107-119, (1962). 


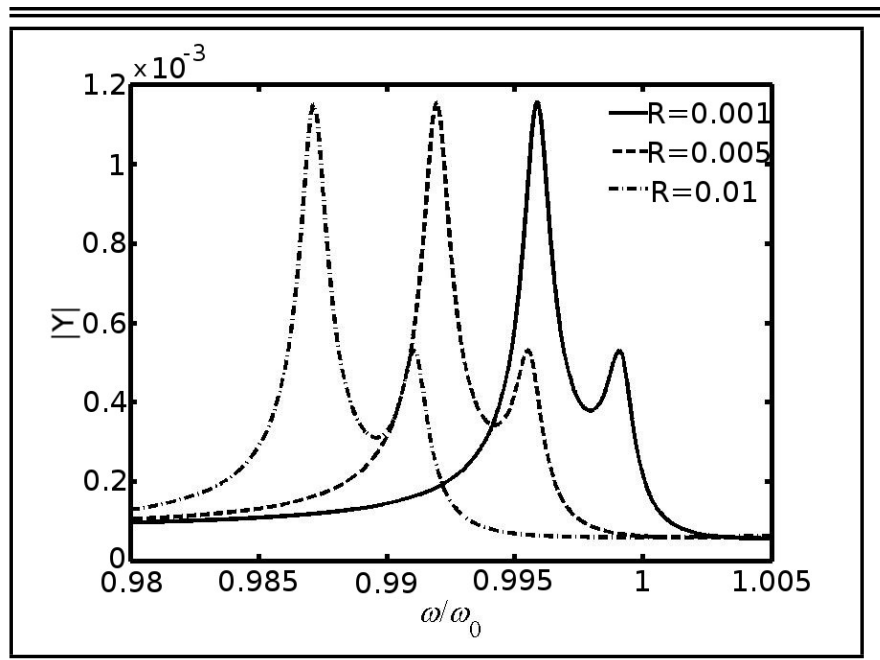

Figure 5. Effect of mass ratio on admittance.

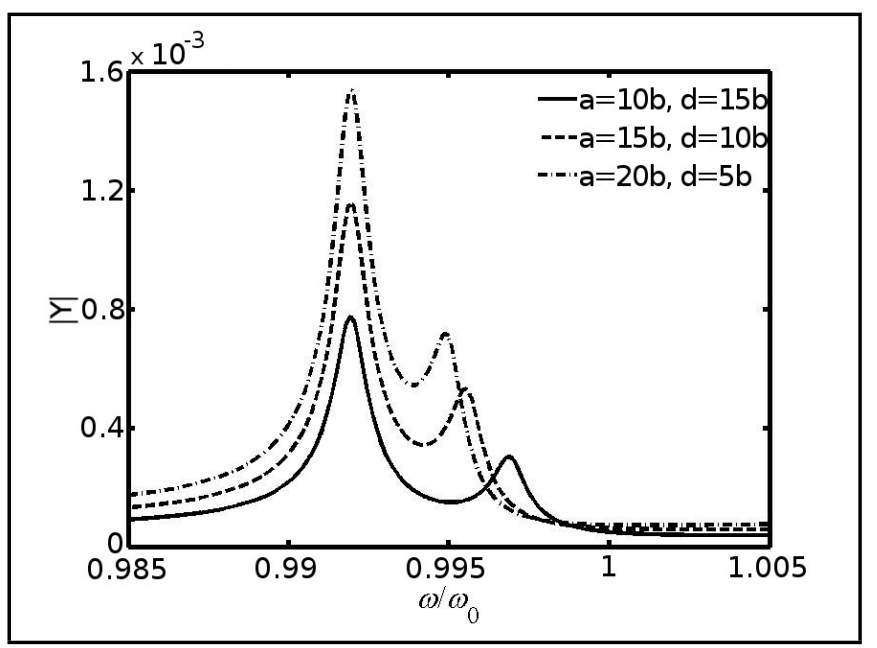

Figure 6. Effect of electrode length on admittance.

3 Mindlin, R. D. High frequency vibrations of piezoelectric crystal plates, Int. J. Solids Struct., 8 (7), 895-906, (1972).

4 Lee, P. C. Y., Syngellakis, S., and Hou J. P. A twodimensional theory for high-frequency vibrations of piezoelectric crystal plates with or without electrodes, J. Appl. Phys., 61 (4), 1249-1262, (1987).

5 Mindlin, R. D. and Lee, P. C. Y. Thickness-shear and flexural vibrations of partially plated, crystal plates, Int. J. Solids Struct., 2 (1), 125-139, (1966).

6 Wang, J., Lee, P. C. Y., and Bailey, D. H. Thickness-shear and flexural vibrations of linearly contoured crystal strips with multiprecision computation, Comput. Struct., 70 (4), 437-445, (1999).

7 Wang, J. and Zhao, W. H. The determination of the optimal length of crystal blanks in quartz crystal resonators, IEEE Trans. Ultrason. Ferroelectr. Freq. Control, 52 (11), 20232030, (2005).

8 Lee, P. C. Y. and Tang, M. S. H. Initial stress field and resonance frequencies of incremental vibrations in crystal resonators by finite element method, Proc. 40th Annual Frequency Control Symposium, (1986).

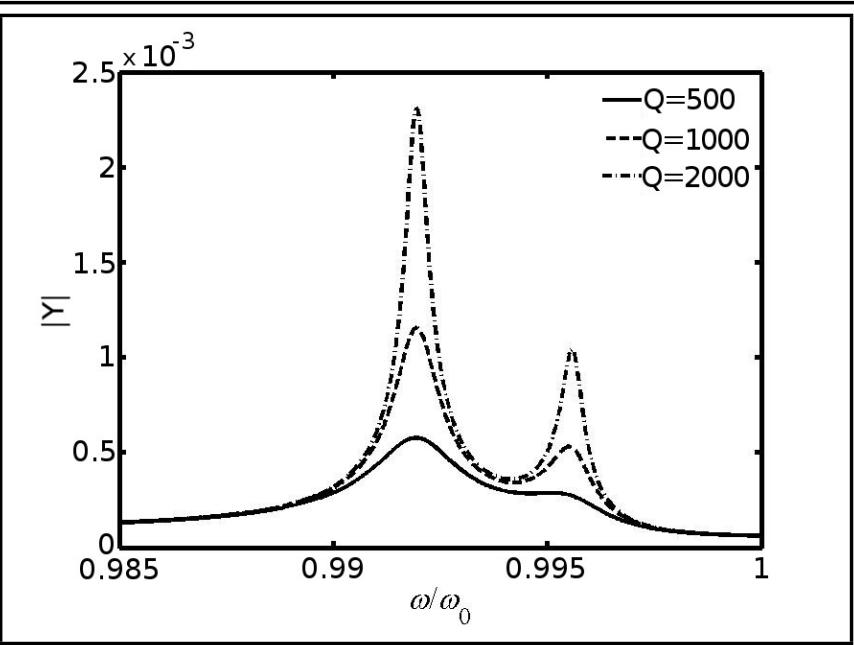

Figure 7. Effect of damping on admittance.

9 Yong, Y. K. and Stewart, J. T. Mass-frequency influence surface, mode shapes, and frequency spectrum of a rectangular AT-cut quartz plate, IEEE Trans. Ultrason. Ferroelectr. Freq. Control, 38 (1), 67-73, (1991).

10 Wang, J. and Yang, J. S. Higher-order theories of piezoelectric plates and applications, Appl. Mech. Rev., 53 (4), 87-99, (2000).

11 Mindlin, R. D. and Spencer, W. J. Anharmonic, thicknesstwist overtones of thickness-shear and flexural vibrations of rectangular, AT-cut quartz plates, J. Acoust. Soc. Am., 42 (6), 1268-1277, (1967).

12 Zhang, C. L., Chen, W. Q., and Yang, J. S. Electrically forced vibration of a rectangular piezoelectric plate of monoclinic crystals, International Journal of Applied Electromagnetics and mechanics, 31 (4), 207-218, (2009).

13 Zhang W. P. Analytical modeling of resistance for AT-cut quartz strips, Proc. IEEE International Frequency Control Symposium, (1998).

14 Zhang, W. P. and Doyle, M. Analytical modeling of resistance for AT-cut quartz strips, in: Yang, J. S. and Maugin, G. A. (Ed.), Mechanics of Electromagnetic Materials and Structures, IOS Press, 147-158, (2000).

15 Wang, J., Zhao, W. H., and Du, J. K. The determination of electrical parameters of quartz crystal resonators with the consideration of dissipation, Ultrasonics, 44 Supplement, e869-e873, (2006).

16 Bottom, V. E. Introduction to Quartz Crystal Unit Design. New York: Van Nostrand Reinhold, (1982).

17 Mindlin, R. D. Thickness-shear and flexural vibrations of crystal plates, J. Appl. Phys., 22 (3), 316-323, (1951). 